\title{
Bi-polar Supernova Explosions
}

\author{
Lifan Wang[, D. Andrew Howell2, Peter Höflich, 3, J. Craig Wheeler \\ Department of Astronomy and McDonald Observatory
}

The University of Texas at Austin

Austin, TX 78712

Received —; accepted —

\footnotetext{
${ }^{1}$ email: lifan@astro.as.utexas.edu

${ }^{2}$ email: howell@astro.as.utexas.edu

${ }^{3}$ email: pah@astro.as.utexas.edu

${ }^{4}$ email: wheel@astro.as.utexas.edu
} 


\begin{abstract}
We discuss the optical spectropolarimetry of several core-collapse supernovae, SN 1996cb (Type IIB), SN 1997X (Type Ic), and SN 1998S (Type IIn). The data show polarization evolution of several spectral features at levels from $0.5 \%$ to above $4 \%$. The observed line polarization is intrinsic to the supernovae and not of interstellar origin. These data suggest that the the distribution of ejected matter is highly aspherical. In the case of SN 1998S, the minimum major to minor axis ratio must be larger than 2.5 to 1 if the polarization is $3 \%$ from an oblate spheroidal ejecta seen edge on. A well-defined symmetry axis can be deduced from spectropolarimetry for the peculiar Type IIn supernova SN 1998S but the Type IIB events SN 1993J and SN 1996cb seem to possess much more complicated geometries with polarization position angles showing larger irregular variations across spectral features; the latter may be associated with large scale clumpiness of the ejecta. The observed degree of polarization of the Type Ic SN 1997X is above 5\%. The data reveal a trend that the degree of polarization increases with decreasing envelope mass and with the depth within the ejecta. We speculate that Type IIB, Type Ib, and Type Ic may be very similar events viewed from different aspect angles.

The high axial ratio of the ejecta is difficult to explain in terms of the conventional neutrino driven core-collapse models for Type II explosions. Highly asymmetric explosion mechanisms such as the formation of bipolar jets during core-collapse may be a necessary ingredient for models of all core-collapse supernovae.
\end{abstract}

Subject headings: stars: individual (SN 1987A, SN 1993J, SN 1996cb, SN1997X, SN 1998S) stars: supernovae - stars: spectroscopy - stars: polarimetry 


\section{Introduction}

Polarimetry probes directly the asphericity of supernova explosions. It is therefore important to the understanding of the supernova explosion mechanism, the pre-explosion environment, and the interaction of the ejecta with their environment.

Only a few supernovae have been observed with polarimetry. SN 1987A represented a breakthrough by providing the first detailed record of the polarimetric evolution (Cropper et al. 1988; Méndez et al. 1988; Jeffery 1991; Höflich 1991; Wang \& Wheeler 1996). SN 1993J also provided a wealth of data (Trammell, Hines, and Wheeler; Doroshenko, Efimov, \& Shakhovskoi 1995; Tran et al 1996). More recently we have begun a program to obtain routine spectropolarimetry on all accessible SNe. We have used mostly the 2.1 and 2.7 meter telescopes at McDonald Observatory. Wang et al (1996) compared broad band polarimetry data obtained at the McDonald Observatory with supernova polarimetry data published before 1996 and found that all Type II in their sample are polarized at about the 1 percent level and that Type Ia are much less polarized, less than 0.2-0.3\%. Two Type Ia, SN 1996X and SN 1999by, are polarized at levels around $0.2 \%$ near optical maximum. Spectropolarimetry of these events revealed numerous features much narrower than typical P-Cygni lines in the flux spectra (Wang, Wheeler, \& Höflich 1997; Howell et al 2000). More data have been acquired since then that confirm that core-collapse events are significantly polarized. The degree of polarization seems to increase with decreasing masses of the stellar envelope, with the highest polarization observed for Type IIB and Type Ib/c events (Wang, Wheeler, Höflich 1999).

In this Letter, we discuss the spectropolarimetry of several core-collapse supernovae obtained at McDonald Observatory (§2). We focus on SN 1996cb (§2.1), SN 1998S (§2.2), and SN 1997X (§2.3), so far the best observed supernovae in our sample, and compare these data with other Type II ( $\$ 2.4)$. The entire polarization data set will be published separately. Here, we discuss the major features which are most relevant to the geometry of the supernova ejecta and to the implications for the core-collapse mechanism. A brief summary is given in $\S 3$.

\section{Observations}

All of the data on SN 1996cb, SN 1997X, and SN 1998S were obtained at the McDonald Observatory using the 2.7 meter telescope or the 2.1 meter telescope. The instrumental polarization of the polarimeter is less than $0.1 \%$, much lower than the polarization of the supernovae. Due to the limited light collecting 
power of our telescopes, we have observed a given supernova with the same configuration for 2-4 nights to get enough photons to reduce statistical noise. Polarized and unpolarized standard stars were observed in all of our observing runs to check the instrumental stability and to correct the position angles of the polarimeter. Multiple sets of data were generally taken with each individual exposure normally 20 to 30 minutes at each waveplate position angle. This is important in our spectropolarimetry since the signals are normally buried in strong noise and independent sets of data are useful in removing spurious features. This procedure also helps to identify features due to cosmic rays which are extremely numerous at the 2.1 meter site. The data for each observing run were independently reduced and then combined to form the final spectra with increased signal to noise ratio. The polarization reduction follows the methods given by Miller, Robinson, \& Goodrich (1988). We have also observed a couple of flux standard stars each observing run to allow for flux calibrations of the supernova spectra. Details of the observations will be given in a forthcoming paper (Wang et al, in preparation).

\subsection{SN 1996cb}

SN 1996cb was discovered pre-maximum on 1996 Dec. 15 (Nakono 1996). SN 1996cb in NGC 3510 is a Type IIB supernova similar to SN 1993J. SNe IIB have lost most of their hydrogen envelopes and have only a tenuous hydrogen layer surrounding them before the explosion. Early spectroscopic data are discussed in a recent paper by Qiu et al. (1999). The supernova reached optical maximum around 1996 Jan. 2. Despite some minor differences pointed out by Qiu et al. (1999), the spectroscopic and photometric behavior of SN 1996cb and SN 1993J are remarkably similar. Such similarity is also shared by another Type IIB SN $1996 \mathrm{~B}$. Both SN 1996cb and SN 1993J showed evidence of clumpiness in the ejecta (Wang \& Hu 1994; Spyromilio 1994; Qiu et al. 1999), and both SNe were positively detected in the radio (Van Dyk et al 1996). Details of SN 1993J can be found in Wheeler \& Filippenko (1996).

Polarimetry data of SN 1993J were obtained around optical maximum. Trammell et al. (1993) reported data taken at the McDonald Observatory on 1993 April 20. These data were analyzed in detail by Höflich et al. (1996) where asymmetric helium cores were assumed to produce the observations. More spectropolarimetry of SN 1993J was reported in Tran et al. (1997). The degree of continuum polarization is an evolving function of time and is around 1.7-2\% around 1993 April 20 - suggesting a highly distorted envelope with major to minor axis ratio $\gtrsim 1.4$ (Höflich, 1991). The supernova must be viewed nearly edge on for an oblate spheroid or pole on for an prolate spheroid according to models of Höflich et al. 
(1996). In contrast to the well defined trace on the Q-U plot observed for SN $1998 \mathrm{~S}$ (cf $\S 2.2$, Fig. 3), the position angles of both SN 1993J and SN 1996cb show large irregular variations across spectral lines. This is consistent with the clumpy ejecta revealed by spectroscopic observations. These must be large scale chemical or ionization clumps. Large numbers of small clumps are unlikely to be efficient polarizer.

The real surprise is that the two supernovae are not only spectroscopically similar but also spectropolarimetrically similar. Figure 1 compares the polarimetry of SN 1996cb obtained on 1996 January 5 with that of SN 1993J taken on 1993 April 26. The SN 1993J data were obtained at the Kitt Peak National Observatory. Sharp polarization changes were seen across the $\mathrm{H} \alpha$ and He 5876 lines. The variation of the polarization is as large as $1.5 \%$ in both supernovae. In addition, data taken after optical maxima show that the polarization of both SN 1993J and SN 1996cb tends to grow with time suggesting that the asymmetries are larger deeper within the ejecta.

The fact that SN 1993J and SN 1996cb show similar spectropolarimetry suggests strongly that the two supernovae shares a similar geometry with a similar orientation to the observer. The nearly identical polarization spectra argues also that, contrary to what has been derived by assuming the emission component of the $\mathrm{H} \alpha$ line is completely de-polarized by the stellar ejecta (Trammell et al 1993; Tran et al 1997), the interstellar polarization is, in fact, negligible for both supernovae. It is worth noting that the pre-maximum spectroscopic data of another Type IIB, SN 1996B, taken at McDonald Observatory again bears striking similarities to those of SN 1993J and SN 1996cb.

A natural question to ask is why these supernovae are so similar despite the fact that they are so highly aspherical and clumpy? The complementary question is whether these supernovae are still Type IIB when viewed at different viewing angles. Our guess is that the fact that they are so similar means that they are likely a select sub-group of supernovae of a more common phenomena. One possibility is that SN Ic, SN Ib, and SN IIB are actually very similar events viewed at different angles. The ejecta may have a tenuous helium-enriched hydrogen disk which is difficult to observe in the polar directions and can only easily be seen close to edge on. The thickness of the hydrogen disk would be related to the relative rate of occurrence of SN Ic, Ib, and IIB. More data, especially spectropolarimetry, will help to create a unified picture for the SN IIB, and perhaps also of SN Ic, and Ib.

At late times, SN 1993J evolved through a remarkable path by showing strong broad hydrogen Balmer emission lines. If the above picture is correct, then we must ask why such broad hydrogen Balmer emission lines have not been detected from other Type Ib/c, or from SN 1996cb. Perhaps SN 1993J had a relatively 
massive circumstellar wind before explosion.

\section{2. $\quad \mathrm{SN} 1998 \mathrm{~S}$}

SN 1998S in NGC 3877 was discovered on 1998 March 2.68 by the Beijing Astronomical Supernova Search program ( $\mathrm{Li}, \mathrm{Li}, \&$ Wan 1998). It was classified as a Type IIn supernova by Filippenko \& Moran (1998) with strong emission lines and no obvious absorption features. It reached optical maximum around March 20. The optical spectra of SN 1998S showed dramatic evolution during the first 3 months. A few days after discovery, the spectra consisted of a blue continuum superimposed with many broad emission lines with no apparent absorption lines. The lines can be identified with emission from $\mathrm{H}, \mathrm{He}$, and C III. The early time spectra are remarkably similar to that of a Wolf-Rayet star (Garnavich, Jha, \& Kirshner 1998). The spectral profiles of all of the emission lines are very similar although they arise from ions with different ionization levels. Leonard et al. (1999) discussed high quality spectropolarimetry of SN 1998S obtained at the Keck II 10-meter telescope on 1998 March 7 UT about two weeks prior to maximum light. They found that the polarization spectrum is characterized by a flat continuum with distinct changes in polarization associated with both a broad (FWZI $\sim 20,000 \mathrm{~km} / \mathrm{sec}$ ) and a narrow $(\mathrm{FWHM}<300 \mathrm{~km} / \mathrm{sec}$ ).

\subsubsection{Spectropolarimetry Data at the McDonald Observatory}

Our spectropolarimetry were obtained using the 2.1 meter telescope of the McDonald Observatory with the Imaging Grism Polarimeter (IGP) on two observing runs each lasting 4 nights. Two sets of data were acquired in the first run, both on the night of 1998 March 30, about 10 days after optical maximum. Each set of data consists of four exposures with exposure time of 20 minutes. Four independent sets of data were obtained during the second run from 1998 April 29 to 1998 May 2. Each set of data again consists of four 20 minutes exposures. All of the data were taken with a slit width of $2^{\prime \prime}$ and have a spectral resolution of about $12 \AA$.

The March 30 and May 1 data were taken at about 10 and 41 days after optical maximum of the supernova, respectively. The latter data were taken just before the light curve began to decline rapidly from the plateau (Garnavich, private communication 1999). The supernova showed dramatic evolution during this period as is shown in Figure 2 where both the spectral and polarimetric behavior had changed dramatically. The March 30 data show an abnormally strong Si II $6355 \AA$ line and a weak H $\alpha$ line on top 
of a blue continuum. The velocity of the absorption troughs of the P-Cygni features is typically $3200 \mathrm{~km}$ $s^{-1}$. The emergence of the strong lines from Fe II, Sc II, Si II indicates that the photosphere has receded into slower moving envelope material. The presence of narrow P-Cygni features indicates that there is slow moving matter, perhaps of circumstellar origin, outside the rapidly expanding ejecta.

Understanding spectropolarimetry may be critical in unraveling the complicated nature of this supernova. As shown in Figure 2. A strong de-polarization is observed across the $\mathrm{H} \alpha$ line, meaning a substantial fraction of the polarization is associated with scattering through material in the vicinity of the supernova rather than merely by interstellar dust particles on the line of sight to the supernova or the scattered light by interstellar dust particles that are very far away from the supernova.

One group of models assumes that the polarization arises from a combination of Thomson and line scattering through the aspherical supernova envelope. This mechanism has been thoroughly explored in several recent studies (Höflich 1995; Höflich et al 1996) and seems to be successful in modeling the spectropolarimetry of SN 1993J. Complete de-polarization by line scattering is assumed in these models. The continua are polarized by electron scattering whereas the de-polarization across spectral lines due to line scattering creates spectral structures across strong P-Cygni lines such as H $\alpha$. Another group of models assumes that the supernovae are associated with an aspherical dusty circumstellar environment which is capable of scattering a significant fraction of the photons from the supernovae (Wang \& Wheeler 1996). Because the dust particles are located at different distances from the center of the supernova, the observer sees photons scattered along different light paths at a specific epoch. A rapidly evolving supernova spectrum will thus produce a rapidly evolving polarimetry spectrum. Note that the degree of polarization is mostly determined by the evolution of the supernovae and to a lesser degree on the scattering properties of the dust and thus it does not necessarily produce higher polarization in the blue than in the red as the conventional perception would suggest. This second mechanism was applied to SN 1987A (Wang \& Wheeler 1996) and produced reasonable fits to the observations. Due to the lack of observational data, it is hard to determine which is a better mechanism in most cases. In many cases it may be a combination of scattering through both the supernova ejecta and the circumstellar dust.

The strong de-polarization across the $\mathrm{H} \alpha$ line in the March 30 spectrum of SN 1998S indicates clearly that resonance scattering through the hydrogen rich envelope is important. Indeed, the pre-maximum spectropolarimetry reported in Leonard et al. (1999) shows a flat polarization spectrum for the continuum which is unlikely to be reproduceable in the circumstellar dust model but is exactly what one would expect 
if the polarization arises from electron scattering within the supernova ejecta.

In terms of the electron scattering model, the March 30 data would be understood in a simple model in which the supernova ejecta and hence the photosphere is highly aspherical, similar to the models that were applied to other Type II supernova such as SN 1987A and SN 1993J. Leonard et al. (1999) found the overall level of polarization to be about 2\% two weeks before maximum. Both our March 30 and May 1 data are taken post optical maximum and the supernova has evolved significantly. Notably, numerous broad absorption lines are detected in our data and polarization spectra have revealed more features than the Keck II data. Our data are unfortunately unable to resolve the narrow $(\mathrm{FWHM}<300 \mathrm{~km} / \mathrm{sec}) \mathrm{H} \alpha$ line detected by the Keck II telescope.

Some important features revealed by the spectropolarimetry can be summarized as follows:

(a) The degree of polarization evolves with time. Polarization variations are observed across strong emission/absorption lines such as $\mathrm{H} \alpha$, Si II, He I, Fe I, and Fe II. Unlike the pre-maximum data (Leonard et al. 1999), the continuum polarization is no longer flat in our post-maximum data. The degree of polarization is the highest in the blue $(\sim 1.6 \%)$ and is the lowest at around $5500 \AA(\sim 0.4 \%) ;(b)$ The position angles of the polarization vector evolved significantly during the two epochs of our observations compared to the earlier Keck II data; (c) A sharp change of polarization position angle by 90 degrees is observed across the $\mathrm{H} \alpha$ line in the May 1 spectrum; (d) the polarization position angle changed by nearly 90 degrees from red to blue in the May 1 spectroscopic data.

\subsubsection{How Large is the Intrinsic Polarization?}

It is important to separate the polarization component due to interstellar dust along the line of sight to the supernova from the components which are intrinsic to the supernova. This is always difficult and depends on certain assumptions of the polarization properties of the emission/absportion lines and the continua. Leonard et al. (1999) were able to separate the $\mathrm{H} \alpha$ lines into a narrow (unresolved) and broad component which show different polarizations. The authors then tried to derive the interstellar polarization by assuming either the narrow or the broad component is intrinsically unpolarized and the observed polarization is due entirely to interstellar polarization. Lacking a proper knowledge of the origin of the narrow and broad components, it is hard to justify which assumption is better.

The maximum degree of interstellar polarization is found to be $9 \times \mathrm{E}(\mathrm{B}-\mathrm{V})$ from observations of 
interstellar polarization in the Galaxy (Serkowski et al 1975). The maximum achievable polarization corresponds to the configuration in which all the dust particles are aligned to the optimum angle for polarization. The circle in Figure 3 shows the limit of allowable interstellar polarization according to the Serkowski relation. It is, however, uncertain because of the uncertainty of the derived extinction.

Certain assumptions must be made in order to estimate the interstellar polarization. We will assess the interstellar polarization via a different approach by relaxing the constraints on the polarization properties of emission lines and assume only that at each specific epoch, the polarization produced by the supernova ejecta has a single polarization position angle independent of wavelength. This can be achieved, for instance, if the polarization is produced purely by electron/dust scattering in the vicinity of the supernova with no large scale chemical inhomogeneities.

The Q-U plot in Fig. 3 is consistent with our assumptions. The polarization at different wavelengths falls roughly on a straight line on the Q-U plot which is typical of the combination of two polarization vectors of which one is nearly constant (like interstellar polarization) while the other highly variable. Under this assumption, the interstellar polarization must lie somewhere along the line drawn by the observed $\mathrm{Q}$ and $\mathrm{U}$ vectors. The allowable areas are marked in Fig. 3 as A and B.

We need theoretical input to determine whether A or B is a more reasonable area for the interstellar polarization. For the 1999 May 1 data, the question is equivalent to asking whether the shorter wavelength or the longer wavelength is more likely to be highly polarized. We believe that the shorter wavelength suffers severe de-polarization due to numerous Fe II, and Sc II lines whereas there are few strong lines at wavelengths longer than $\mathrm{H} \alpha$ and therefore the longer wavelength side is more likely to have higher polarization. This limits the possible interstellar component to area A. In the subsequent discussion we will take $\mathrm{Q} \sim 0.7 \%$ and $\mathrm{U} \sim-1.9 \%$ for the interstellar polarization. Note also our determination of the interstellar polarization yields a position angle of -35 degrees for the interstellar polarization.

This determination of the interstellar polarization leads to intrinsic polarization of the supernova of around $1 \%$ for the March data and over 3\% a month later. To produce such a high degree of polarization for density profiles which resemble those of SNe II, the major/minor axis ratios must be larger than 1.2 to 1 and 2.5 to 1 for the early and late observations, respectively, if the ejecta distribution is an oblate spheroid viewed edge on. Any other viewing angle $\Theta$ will require larger axis ratios because the polarization scales as $\approx P($ equatorial $) \sin ^{2} \Theta$. Once again, we note that the polarization increases with time suggesting greater distortions the deeper one goes in the ejecta. The degree of polarization is extraordinarily large and clearly 
shows a bi-polar structure for the ejecta.

Another Type IIn, SN 1994Y, was also observed in our program close to optical maximum and showed similar behavior to SN 1998S (Wang et al. 1996). The degree of polarization variation across the $\mathrm{H} \alpha$ line is larger than $1.5 \%$ in that case, and by using the same method for separating the interstellar polarization, the intrinsic polarization can be as large as 3\%. This is consistent with SN 1998S. These are the only two Type IIn supernovae with polarimetry observations so far. We note that, despite their extraordinarily flat light curves, Type IIn supernovae are probably an extreme class of objects which have lost most of their massive envelope and the ejecta mass can be less than $1 \mathrm{M}_{\odot}$ (Chugai, \& Danziger 1994). This is consistent with the Wolf Rayet features of SN 1998S and of its interpretation as a Wolf Rayet star by Gerardy et al. (1999).

\subsection{Type Ic SN $1997 X$}

SN 1997X is one of the best observed supernovae in terms of polarimetry. The observed total degree of polarization is as high as 7\%. A significant fraction of the polarization may be of interstellar origin. The polarized spectra do show clear spectral features across the strong He I 5876, 6678 lines (Wang, Wheeler, Höflich 1997). Although it is hard to separate the intrinsic and interstellar polarization, it is also clear that a large faction of the of the polarization must be associated with the supernova ejecta. Fig. 4 shows the time evolution of the continuum polarization. A polarization level of at least $4 \%$ can be attributed to an asymmetric ejecta with axis ratios larger than 3 to 1 for oblate spheroids.

\subsection{Other Type II supernova}

We have observed a few normal Type II plateau supernovae which are believed to have more massive stellar envelopes. The degree of polarization is not as large as those of SN 1987A (at late times), SN 1993J, SN 1996cb, and SN 1997X and is typically around 0.5-1.5\%. These supernovae and the detected levels of polarization are: SN 1995H, 1\%; SN 1995V, 1.5\%; SN 1996W, 0.7\% which corresponds to deviations from symmetry between 10 to $30 \%$. We expect relatively low polarization because of the damping effect of the hydrogen envelope. Even these moderate levels of polarization for SN IIP suggest a strong asymmetry deep

within the ejecta. So far, we have observed no exceptions to the statement that all core-collapse supernovae are substantially polarized and hence substantially asymmetric. The recent supernova SN 1999em was probably a supernova with a massive envelope. Pre-maximum polarimetry at the Keck II telescope showed 
no intrinsic polarization down to $0.1 \%$ suggesting either a spherical ejecta or a nearly pole-on viewing angle (Leonard, Filippenko \& Chornok 1999). Alternatively, the polarization could also be suppressed if the density slope is very steep as indicated by the small differential Doppler shifts of the lines of the Balmer series (Höflich 1995). This null result may thus be due to the early epoch of observation. It will be interesting to obtain post-maximum data to probe deeper into the ejecta to regions more directly related to the explosion mechanism.

\section{Discussion}

These polarization observations reveal that core-collapse supernovae are generally polarized and are highly aspherical. The degree of polarization evolves with time and generally increases after optical maximum. The polarization is also anti-correlated with the mass of the remaining hydrogen shell. The trend is that highest polarizations are observed for supernovae that have lost most of their hydrogen envelope before explosion. This trend is illustrated by the sequence Type II SN 1987A, Type IIn SN 1994Y and SN 1998S, Type IIB SN 1993J and 1996cb, and Type Ic SN 1997X. From their relatively large variations of the polarization position angles across spectral lines, Type IIB events seem to possess not only large global asymmetry but also strong large scale chemical or ionization clumps.

Polarization as large as $4 \%$ along a constant orientation axis requires a strongly bi-polar departure from spherical symmetry. How was such a geometry produced? Does the spectropolarimetry reveal an intrinsically aspherical supernova explosion explosion? One possibility might involve massive stellar disks surrounding the supernova so that even though the explosion is spherical, the ejecta/disk interaction could distort the photosphere. The increasing degree of polarization after optical maximum observed in SN 1987A, SN 1993J, SN 1996cb, SN 1997X, and SN 1998S strongly favors an aspherical explosion.

Both the temporal evolution and the dependency on ejecta mass of the polarization are consistent with aspherical explosions. The asphericity is expected to be the largest near the center of the ejecta which is normally revealed only by observations past optical maximum. The asymmetry is also expected to have the largest effect on the ejecta geometry for bare-core progenitors such as those for Type $\mathrm{Ib} / \mathrm{c}$ since a massive hydrogen envelope tends to smear out the asphericity.

These observations argues strongly against the conventional picture for Type II supernovae explosions. It is hard to imagine that neutrino-driven explosions can produce such large asymmetries throughout the 
ejecta. We note that the neutrino-driven explosion models have so far failed to produce robust explosions. To produce the bi-polar structure we observe with the polarimetry, a strong asymmetry must be imposed and maintained for a time substantially long compared to the dynamical time of the progenitor. This criterion can be satisfied by the production of a sustained jet from deep within the core. The recent jet-driven explosion models by Khokhlov et al. (1999) and MacFadyen \& Woosley (1999) provide a more promising approach to accounting for the polarization. We note that since the polarization is ubiquitous these jets must routinely arise from neutron stars, not just in the rare events that produce explosions and black holes. Asymmetric jets could provide both the bi-polar ejecta and a kick to the neutron star (Khokhlov et al. 1999). On the contrary, asymmetric neutrino emission could deliver a kick to a pulsar, but it is unlikely to generate the bulk bi-polar nature of the ejecta that we deduce.

We thank Alex Filippenko and Doug Leonard for useful discussions and for providing data before publication. This work is supported in part by NSF Grant 9818960, by a grant from the Texas Advanced Research Program and by NASA through grants LSTA-98-02 and HF-01085.01-96A from the Space Telescope Science Institute which is operated by the Association of Universities for Research in Astronomy, Inc., under NASA contract NAS 5-26555. 


\section{REFERENCES}

Chugai, N. N., \& Danziger, I. J. 1994, MNRAS, 268, 173

Cropper, M., Bailey, J. A., McCowage, J., Cannon, R. D., Couch, W. J., Walsh, J. R., Straede, J. O., \& Freeman F. 1988, MNRAS, 231, 695

Doroshenko, V. T., Efimov, Y. S., \& Shakhovskoi, N. M. 1995, Astrophys. Lett., 21, 513

Filippenko, A. V., \& Moran E. C. IAUC, 6829

Garnavich, G., Jha, S., \& Kirshner, R. 1998, IAUC, 6832

Gerady, C. L., Fesen, R. A., Höflich, P., \& Wheeler, J. C. 1999, ApJ, in press

Hö̈lich, P. 1991, A\&A 246481

Höflich, P. 1995, ApJ, 440, 821

Höflich, P., Wheeler, J. C., Hines, D. C., \& Trammell, S. R. 1996, ApJ, 459, 307

Howell, D. A. et al 1999, in preparation

Leonard, D. C., Filippenko, A. V., Bath, A. J., \& Matheson, T. 1999, submitted to ApJ (astro-ph/9908040).

Leonard, D. C., Filippenko, A. V. \& Chornock, R. T. 1999, IAUC, 7305

Li, W.-D., Li, C., \& Wan, Z. 1998 IAUC, 6829

McFadyen, A. I., \& Woosley, S. E. 1999, ApJ, 524, 262

Mendez, M., Clocchiatti, A., Benvenuto, O. G., Feinstein, C., \& Marraco, H. G. 1988, ApJ, 334, 295

Miller, J. S., Robinson, L. B., \& Goodrich, R. W. 1988, in Instrumentation for Ground-Based Astronomy, ed. L. B. Robinson (New York: Springer), p. 157

Nakano, S. 1996, IAUC 6514

Qiu, Y., Li, W., Qiao, Q., \& Hu, J. 1999, AJ, 117, 736

Serkowski, K., Mathewson, D. S., \& Ford, V. L. 1975, ApJ, 196, 261

Spyromilio, J. 1994, MNRAS, 266, 61

Trammell, S. R., Hines, D. C., \& Wheeler, J. C. 1993, ApJ, 414, L21

Tran, H.D., Filippenko, A.V., Schmidt, G.D., Bjorkman, K.S., Jannuzi, B.T., \& Smith, P.S. 1997, PASP, 109,489 
Van Dyk, S. D., Sramek, R. A., Montes, M. J., Weiler, K. W., \& Panagia, N. 1996, IAUC 6528

Wang, L., \& Hu, J. Y. 1994, Nature, 369, 380

Wang, L., \& Wheeler, J. C. 1996 ApJ, 462, L27

Wang, L., Wheeler, J. C., \& Höflich, P. 1997, ApJ, 476, L27

Wang, L., Wheeler, J. C., \& Höflich, P. 1999, in SN 1987A: Ten Years After, ed. M. M. Phillips \& N. Suntzeff (Provo: ASP), in press

Wang, L., Wheeler, J. C., Li, Z., \& Clocchiatti, A. 1996, ApJ, 467, 435

Wheeler, J. C., \& Filippenko, A. V. 1996, in Supernovae and Supernova Remnants, ed. R. McCray and Z. Wang (Cambridge, Cambridge U. P.), p. 241 


\section{Figure Captions}

Fig. 1... The total flux and polarization spectra of SN 1996cb are compared to those of SN 1993J.

Fig. 2... Spectropolarimetry of SN 1998S. The left column gives the total flux spectrum, the percent polarization and the polarization angle for the data of 1998 March 30, respectively and the right column gives the corresponding data for 1998 May 1.

Fig. 3... The Spectropolarimetry of SN 1998S on the Q-U plane for 1998 March 30 (left panel) and 1998 May 1 (right panel). The outer circles are the limit of the interstellar polarization from the extinction. The premaximum data from Leonard et al. (1999) is labeled by Keck II data. The circles labeled A and B represent the two choices for the ISM, of which we prefer A (see text). The small colored circles in the May 1 data are from $6397 \AA$ to $6802 \AA$ in the vicinity of the $\mathrm{H} \alpha$ line.

Fig. 4... The evolution of the continuum polarization (Q, U, and total polarization P), for SN 1997X obtained by averaging from $5000 \AA$ to $6000 \AA$. Note the large change with time which must be intrinsic to the supernova and suggests a polarization of order $4 \%$. 


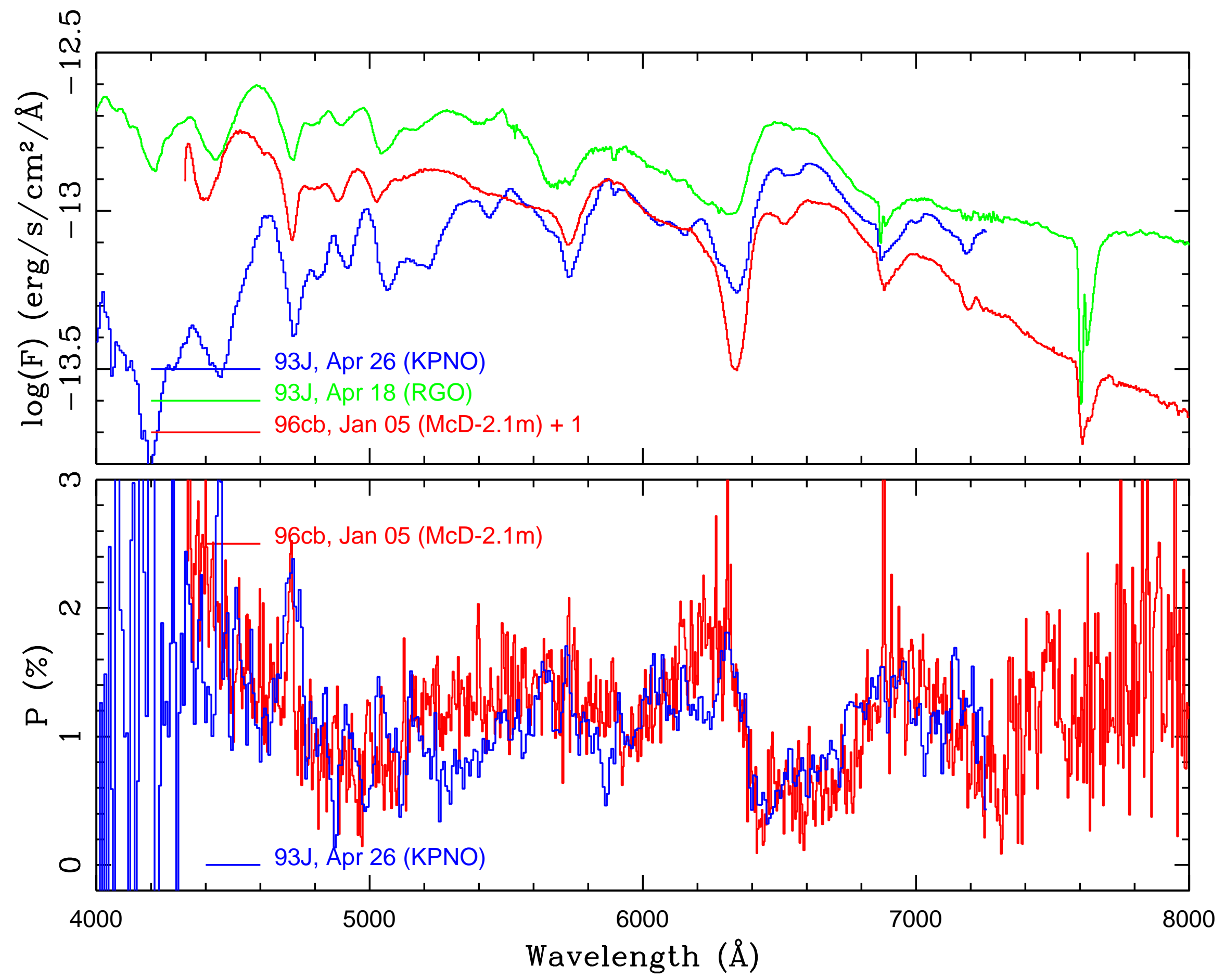




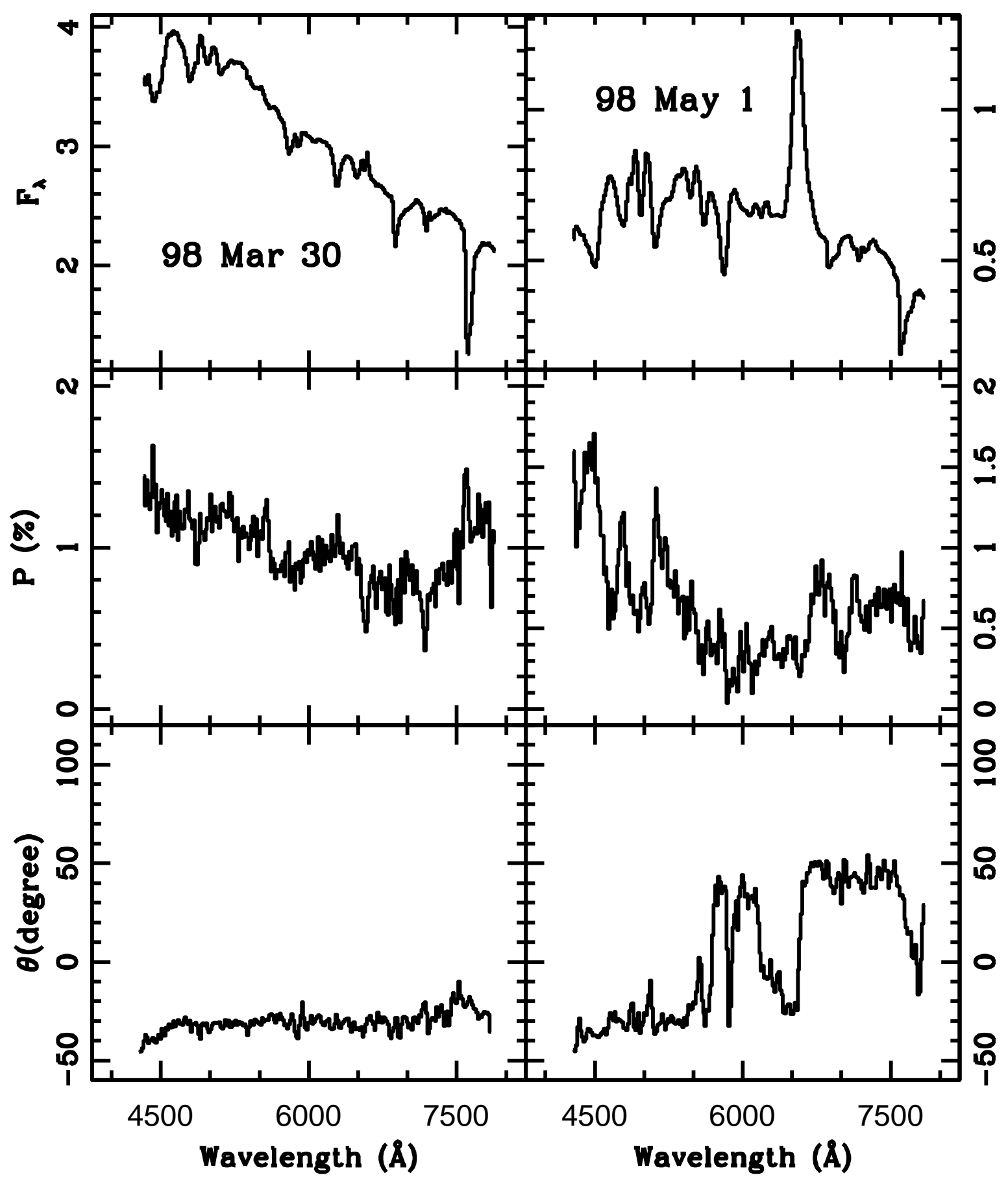




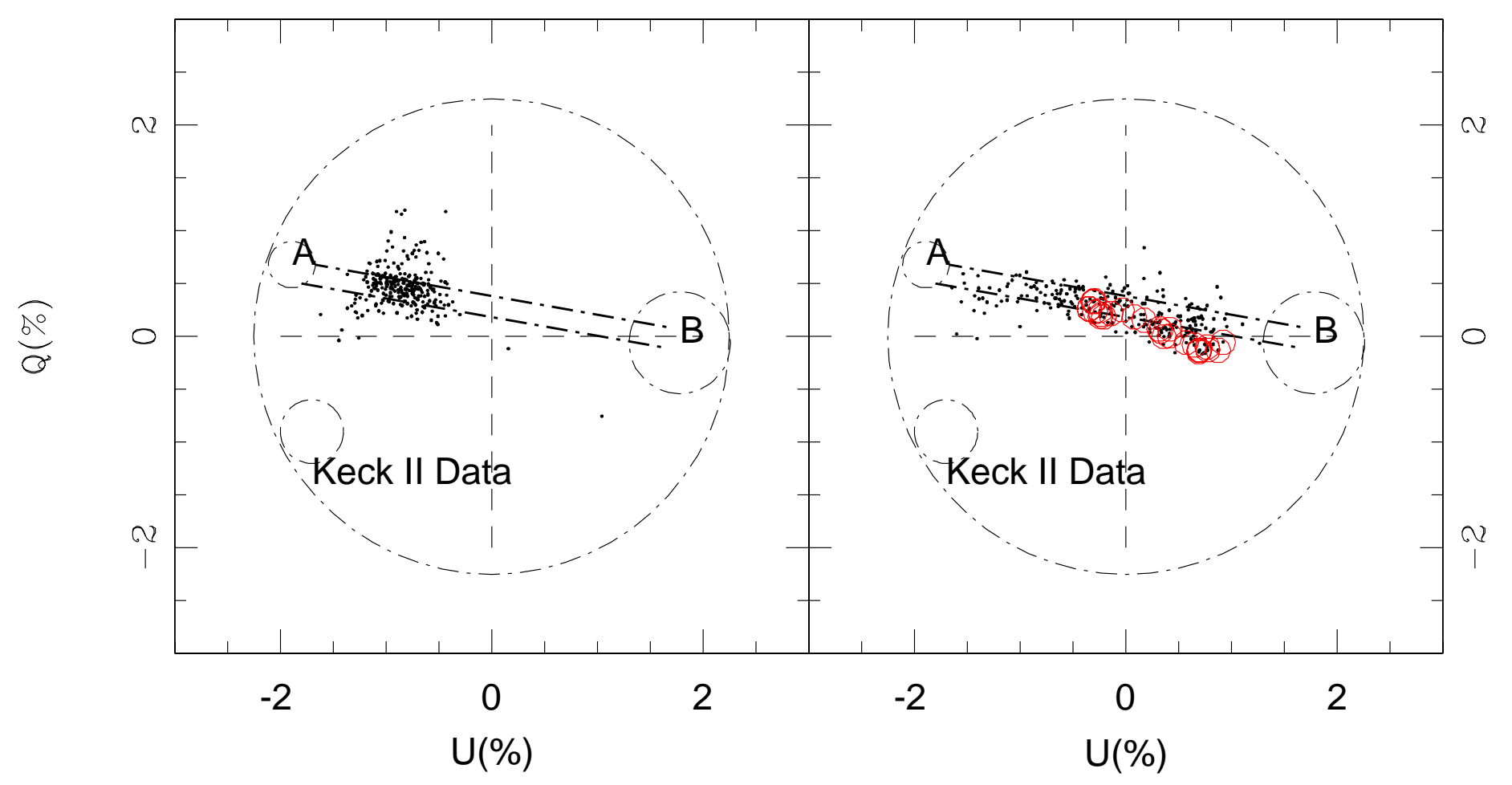




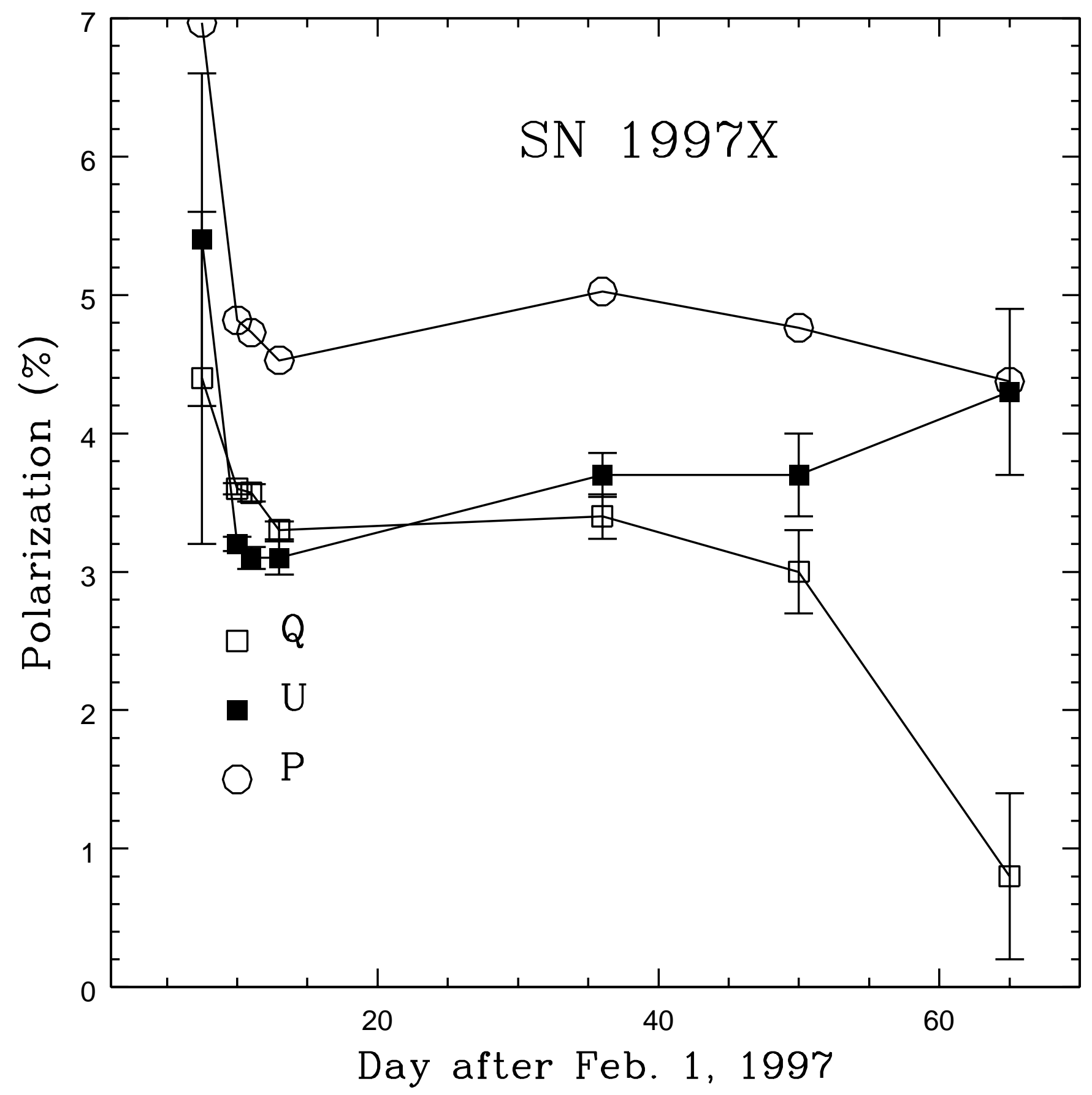

